Instituto Internacional de Investigación y Desarrollo Tecnológico Educativo INDTEC, C.A. DOI: https://doi.org/10.29394/Scientific.issn.2542-2987.2020.5.17.2.39-55

OAI-PMH: http://www.indteca.com/ojs/index.php/Revista Scientific/oai

Artículo Original / Original Article

\title{
Impacto en la función docente y el proceso académico por el cambio a la modalidad virtual
}

\author{
Autores: César Raúl Méndez Carpio \\ Universidad Católica de Cuenca, UCACUE \\ cmendezc@ucacue.edu.ec \\ Cuenca, Ecuador \\ https://orcid.org/0000-0003-0582-0107 \\ Natalia Adriana Palacios Montero \\ Universidad Técnica Particular de Loja, UTPL \\ npalacios@napalogistic.com \\ Cuenca, Ecuador \\ https://orcid.org/0000-0001-6625-2559
}

\section{Resumen}

La situación actual en el mundo ha desarrollado nuevas formas de ver la vida y de valorar hechos que han tomado relevancia en los últimos meses, específicamente desde marzo del año 2020 en que la pandemia del Covid-19 tomó fuerza en el Ecuador y el resto del planeta. Ha desatado una crisis cultural que impacta de forma creciente en todos los campos de acción y se destaca en la función docente y el cambio en el proceso académico. La investigación pretende analizar el impacto que ha tenido el paso emergente de la modalidad de estudios presenciales a la modalidad virtual en la tarea diaria del docente y su repercusión en la ejecución de su gestión; así mismo centrar la atención en el aislamiento social y los procesos de educación alternativa que denotan su estudio. Se considera un enfoque mixto basado en el método de revisión bibliográfica apoyado en la aplicación de encuestas estructuradas al personal docente que ha vivenciado el cambio. Se pudo notar que tanto el personal docente como los estudiantes, no estuvieron preparados para adoptar de manera urgente e inmediata la realidad presente lo que ocasionó cambios estructurales en su forma de impartir y recibir información, así como afecciones en el proceso académico resultante.

Palabras clave: crisis cultural; educación alternativa; aislamiento social.

Cómo citar este artículo:

Méndez, C., \& Palacios, N. (2020). Impacto en la función docente y el proceso académico por el cambio a la modalidad virtual. Revista Scientific, 5(17), 39-55, e-ISSN 2542-2987. Recuperado de: https://doi.org/10.29394/Scientific.issn.2542-2987.2020.5.17.2.39-55

Fecha de Recepción: 09-03-2020
Fecha de Aceptación:

30-05-2020
Fecha de Publicación: 05-08-2020 
OAI-PMH: http://www.indteca.com/ojs/index.php/Revista_Scientific/oai

Artículo Original / Original Article

\title{
Impact on the teaching function and the academic process due to the change to the virtual modality
}

\begin{abstract}
The current situation in the world has developed new ways of looking at life and evaluating events that have become relevant in recent months, specifically since March 2020, when the Covid-19 pandemic took hold in Ecuador and the rest of the world. planet. It has unleashed a cultural crisis that increasingly impacts all fields of action and stands out in the teaching function and the change in the academic process. The research aims to analyze the impact that the emerging step from the modality of face-to-face studies to the virtual modality has had on the daily work of the teacher and its repercussion in the execution of its management; likewise focus attention on social isolation and alternative education processes that denote their study. It is considered a mixed approach based on the bibliographic review method supported by the application of structured surveys to teaching staff who have experienced the change. It could be noted that both the teaching staff and the students were not prepared to urgently and immediately adopt the current reality, which caused structural changes in the way they impart and receive information, as well as affects the resulting academic process.
\end{abstract}

Keywords: cultural crises; alternative education; social isolation.

How to cite this article:

Méndez, C., \& Palacios, N. (2020). Impact on the teaching function and the academic process due to the change to the virtual modality. Revista Scientific, 5(17), 39-55, e-ISSN: 2542-2987. Recovered from: https://doi.org/10.29394/Scientific.issn.2542-2987.2020.5.17.2.39-55

Date Received: 09-03-2020
Date Acceptance:

30-05-2020
Date Publication: 05-08-2020 


\section{Introducción}

La función docente y el proceso académico vienen de la mano desde que el hombre decide formar parte de la sociedad y por tanto sus actividades han sido motivo de distintas teorías y etapas que han volcado la educación hacia el logro del rendimiento académico conseguido a través de distintas técnicas didácticas y pedagógicas con el fin de desarrollar destrezas y aptitudes en los estudiantes y su formación.

El conocimiento al estar en proceso de internalización, genera un nivel de rendimiento que es valorado en las distintas etapas educativas a través de las actividades de evaluación generando, promedios académicos que resultan en, durante y al final de un seguimiento como un buen o mal rendimiento académico.

El rendimiento académico es una problemática que preocupa a toda la comunidad educativa, siendo hoy en día motivo de estudio de varios procesos investigativos en el Ecuador, Latinoamérica y es preocupación constante en todos los continentes. Los docentes deben estar conscientes de su papel fundamental, que resulta ser el mediador del hecho académico apoyado en los padres de familia y/o representantes buscando constantemente la aplicación de nuevas estrategias metodológica para conseguir el desarrollo académico de calidad en función de una formación integral de sus estudiantes.

Varios autores coinciden, analizan y sostienen que el aprendizaje de los estudiantes depende de un buen rendimiento académico promovido por los métodos didácticos que aplica el docente y lo conseguido por los estudiantes. Para Martínez-Otero (1997), el rendimiento académico es: "el producto que da el alumnado en los centros de enseñanza y que habitualmente se expresa a través de las calificaciones escolares" (pág. 34). Aunque para Angarita y Cabrera (2000): "[...] un alto rendimiento académico no es suficiente para garantizar prosperidad, prestigio, ni felicidad en la vida [...]" (pág. 4).

Un criterio con más concordancia en este estudio es el de Caballero, 
OAI-PMH: http://www.indteca.com/ojs/index.php/Revista_Scientific/oai

Artículo Original / Original Article

Abello y Palacio (2007): quienes coinciden que el rendimiento académico debe enfocarse en el cumplimiento de todos los objetivos, logros y metas planteados en el plan de estudios de cada asignatura; este criterio se enfoca al aspecto en que los estudiantes deben mantener una adecuada valoración de su rendimiento.

Lo que se menciona, se ha hecho transversal en la presencialidad vivida durante el desarrollo de la función docente y el acercamiento frente a los procesos de comunicación con sus estudiantes, manteniéndose los roles bien delimitados que han servido de puente a la educación tradicional en las instituciones que mantenían la educación cara a cara. Considerando el cambio social dado por la pandemia que inmovilizó el mundo y en correspondencia con Dolores-Martínez y Chávez (2015): “los contenidos digitales mediados por plataforma tecnológica, pueden considerarse como una herramienta para ser usada en los procesos educativos tal y como queremos proyectarlos, representa un desafío e implica modificar paradigmas y formas de abordar el conocimiento [...]" (pág. 378).

Hoy en día tanto el rol del docente como del estudiante han dado un giro de 180 grados y se considera un cambio radical en metodología, recursos, políticas, procedimientos, entre otros, producto de dicho cambio social y del aislamiento domiciliario impuesto que ha volcado todo el sistema a la modalidad virtual estando o no preparados para afrontarlo.

En referencia con el contexto de la complejidad en la educación, Martínez, Martínez, Martínez y León (2018), asevera que se deben cultivar el liderazgo a través de características propias de un comunicador como:

[...] Su responsabilidad con los seguidores y la organización, así como esa capacidad para la escucha activa, el manejo de los mensajes entre otras, representa entonces el desafío de trabajar sobre la idea de contar con líderes en todos los niveles orientando u orientados tras una clara e internalizada Visión y la creación de futuros, que configuren diseños de organizaciones acordes, estimulen la cooperación y el trabajo 
en equipo [...] (págs. 206-207).

Desde este escenario, los docentes deben mantener la figura de líderes independientemente en la forma en que se imparta las clases y con mucha más agudeza cuando se trata de una modalidad que separa las distancias y puede dividir el mensaje entre los componentes de la comunicación.

Se deben considerar teorías y preceptos del docente como líder y como responsable de los procesos educativos en la modalidad virtual ya que en este proceso se integran materiales y recursos didácticos multimedia desarrollados con la suficiente capacidad para guiar los aprendizajes a través de la interacción comunicativa entre el docente y sus estudiantes. Por esta razón se ha vuelto imprescindible el uso de las Tecnologías de Información y Comunicación (TIC) que al respecto Flores y Garrido (2019), explican que:

Son catalizadores que transforman y modifican los actuales escenarios de aprendizaje. Con ello emerge la necesidad de fortalecer las competencias de los docentes en los diferentes niveles de educación que contribuyan en el uso pedagógico de las TIC, propiciando motivación y dinamizando los procesos de enseñanza-aprendizaje que se vinculan a los requerimientos de la sociedad del conocimiento (pág. 46).

Si se modifican los escenarios entonces, pasan a un primer plano el conocimiento y dominio de herramientas informáticas que faciliten la consecución de los aprendizajes y el desarrollo de destrezas en los educandos. Es así que, al momento de actuar como docente, hay que saber seleccionar de la gran gama de aplicaciones existentes, cuáles serán las más efectivas y acorde a los contenidos para poderlas consumir.

Los docentes frente a estos cambios deben considerar la adecuación de sus perfiles enfocándose en la autonomía de sus actos, disciplina consciente, procedimientos de innovación y auto superación personal como premisas de su nueva motivación y desarrollo profesional siendo muy 
OAI-PMH: http://www.indteca.com/ojs/index.php/Revista_Scientific/oai

Artículo Original / Original Article

importante repensar los atributos de la profesión con miras a transformar la actitud y la aptitud deseada, conforme a Castellanos y Castro (2018): "esto infiere un escenario actual donde la tecnologización provoca transformaciones en cuanto a la manera de concebir el conocimiento, constituir equipos de alto desempeño, comunicarse con los demás, originar nuevas maneras de aprendizaje, ocupar tiempo en adquirir habilidades tecnológicas" (pág. 101).

En la investigación presente, se ha considerado analizar entonces, el impacto que ha tenido el paso emergente de la modalidad de estudios presenciales a la modalidad virtual desde distintos ámbitos de la función docente y su repercusión en la ejecución del proceso académico a través de la caracterización de criterios sobre la gestión docente basado en el rendimiento académico de los estudiantes y el tratamiento de las competencias digitales que le permitirán afrontar la crisis y bajar el nivel de impacto generado, proponiendo el uso de aplicaciones TIC para facilitar la función docente y facilitar el proceso académico.

\section{Metodología (Materiales y métodos)}

La metodología aplicada, tiene un enfoque cualitativo apoyada en los preceptos del método fenomenográfico, que ha sido considerado para una mejor comprensión de la situación vivida por los docentes y estudiantes en el proceso de transición estudiado. Además, el estudio se apoyó en una investigación bibliográfica sobre la modalidad virtual y sus preceptos, así como la aplicación de una encuesta estructurada a la muestra intencional de 120 docentes de distintos niveles académicos que laboran en instituciones de prestigio en la ciudad de Cuenca y que cuentan en su gran mayoría con una trayectoria académica estable en la función docente.

Las encuestas se desarrollaron y fueron aplicadas a través de un formulario en línea con direccionamiento a correos electrónicos notados en una base de datos de profesionales de la educación en los niveles de 
educación general básica en un $33,33 \%$, bachillerato en un $38,67 \%$ y educación superior en el $28 \%$ de los encuestados.

\section{Resultados (análisis e interpretación de los resultados)}

Al analizar el impacto que ha tenido el paso emergente de la modalidad de estudios presenciales a la modalidad virtual en la función docente y su repercusión en el proceso académico, las características específicas de dicho impacto fueron delimitadas con la aplicación de la encuesta de información estructurada a docentes de distintos niveles de educación básica, bachillerato y superior, siendo un $93,3 \%$ de docentes de la muestra intencional así como a una parte a personal del Departamento de Consejería Estudiantil (DECE) en un total muestral del $6,7 \%$.

Se mereció seccionar la investigación considerando las dificultades encontradas para adaptar el currículo a la modalidad virtual y priorizando distintos parámetros que direccionaron la estructura de las preguntas en las siguientes dimensiones: adaptación y apoyo al proceso de cambio, alfabetización y destrezas en la modalidad virtual, distribución de servicios de ancho de banda, cumplimiento de objetivos en el proceso de enseñanza aprendizaje, actividades de repercusión en la salud mental, física y el uso adecuado del tiempo, como se puede observar en la tabla 1.

Tabla 1. Dificultades encontradas en la gestión docente y el proceso académico (Resultado del estudio).

\begin{tabular}{|l|c|c|}
\hline \multicolumn{2}{|c|}{ Dificultades encontradas para adaptar el currículo a la modalidad virtual } \\
\hline \multicolumn{1}{|c|}{ ADAPTACIÓN Y APOYO AL PROCESO DE CAMBIO } & SI & NO \\
\hline $\begin{array}{l}\text { Se considera Ud. lo suficientemente preparado para el cambio tecnológico a la } \\
\text { modalidad virtual o e-learning. }\end{array}$ & $45,0 \%$ & $55,0 \%$ \\
\hline Evidencia Ud. la falta de equipo tecnológico (hardware software) & $65,0 \%$ & $35,0 \%$ \\
\hline Evidencia Ud. la falta de capacitación. & $70,0 \%$ & $30,0 \%$ \\
\hline $\begin{array}{l}\text { Se muestran insuficientes las políticas impuestas por parte de los organismos de } \\
\text { gobierno. }\end{array}$ & $78,3 \%$ & $21,7 \%$ \\
\hline Considera Ud. insuficiente el apoyo por parte de la institución donde labora. & $25,0 \%$ & $75,0 \%$ \\
\hline
\end{tabular}


Instituto Internacional de Investigación y Desarrollo Tecnológico Educativo INDTEC, C.A.

DOI: https://doi.org/10.29394/Scientific.issn.2542-2987.2020.5.17.2.39-55

OAI-PMH: http://www.indteca.com/ojs/index.php/Revista_Scientific/oai

Artículo Original / Original Article

\begin{tabular}{|c|c|c|}
\hline $\begin{array}{l}\text { Se muestran insuficientes las políticas informáticas por parte de las autoridades } \\
\text { académicas institucionales }\end{array}$ & $30,0 \%$ & $70,0 \%$ \\
\hline ALFABETIZACIÓN Y DESTREZAS EN LA COMUNICACIÓN VIRTUAL & SI & NO \\
\hline $\begin{array}{l}\text { Se muestra insuficiente conocimiento del manejo de software para video } \\
\text { conferencias. }\end{array}$ & $35,0 \%$ & $65,0 \%$ \\
\hline $\begin{array}{l}\text { Se muestra insuficiente conocimiento del manejo de software de trabajo } \\
\text { colaborativo. }\end{array}$ & $51,7 \%$ & $48,3 \%$ \\
\hline $\begin{array}{l}\text { Se muestra insuficiente conocimiento de herramientas web } 2.0 \text { para desarrollo de } \\
\text { presentaciones. }\end{array}$ & $53,3 \%$ & $46,7 \%$ \\
\hline $\begin{array}{l}\text { Se muestra insuficiente conocimiento de herramientas web } 2.0 \text { para colaboración } \\
\text { sincrónica. }\end{array}$ & $58,3 \%$ & $41,7 \%$ \\
\hline $\begin{array}{l}\text { Se muestra insuficiente conocimiento de herramientas web } 2.0 \text { en software para } \\
\text { evaluación en línea. }\end{array}$ & $56,7 \%$ & $43,3 \%$ \\
\hline $\begin{array}{l}\text { Se muestra insuficiente conocimiento de herramientas web } 2.0 \text { en software para } \\
\text { pizarras digitales. }\end{array}$ & $86,7 \%$ & $13,3 \%$ \\
\hline $\begin{array}{l}\text { Se muestran dificultades en la expresión frente a una cámara durante las clases } \\
\text { virtuales }\end{array}$ & $20,0 \%$ & $80,0 \%$ \\
\hline $\begin{array}{l}\text { Se muestran dificultades al mantener la comunicación con los estudiantes al otro } \\
\text { lado de la línea. }\end{array}$ & $45,0 \%$ & $55,0 \%$ \\
\hline Se muestra insuficiente control disciplinario con los estudiantes de la conferencia. & $33,3 \%$ & $66,7 \%$ \\
\hline DISTRIBUCIÓN DE SERVICIOS DE ANCHO DE BANDA & SI & NO \\
\hline $\begin{array}{l}\text { Considera Ud. que son suficientes los anchos de banda contratados en los } \\
\text { hogares para la comunicación en línea }\end{array}$ & $71,7 \%$ & $28,3 \%$ \\
\hline $\begin{array}{l}\text { Considera Ud. que ha impactado la cantidad de usuarios en el hogar frente al } \\
\text { ancho de banda contratado. }\end{array}$ & $88,3 \%$ & $11,7 \%$ \\
\hline $\begin{array}{l}\text { Considera Ud. que son suficientes los servicios de ancho de banda ofrecidos por } \\
\text { las empresas proveedoras en su localidad. }\end{array}$ & $58,3 \%$ & $41,7 \%$ \\
\hline $\begin{array}{l}\text { CUMPLIMIENTO DE OBJETIVOS EN EL PROCESO DE ENSEÑANZA } \\
\text { APRENDIZAJE }\end{array}$ & SI & NO \\
\hline Evidenció Ud. la falta de contenido científico para dictar sus clases. & $15,0 \%$ & $85,0 \%$ \\
\hline Considera Ud. insuficiente el material de apoyo didáctico & $33,3 \%$ & $66,7 \%$ \\
\hline $\begin{array}{l}\text { Considera Ud. insuficiente al material académico en relación con la asignatura que } \\
\text { imparte. }\end{array}$ & $33,3 \%$ & $66,7 \%$ \\
\hline $\begin{array}{l}\text { Se muestran dificultades en el avance de los contenidos en función con la } \\
\text { planificación. }\end{array}$ & $50,0 \%$ & $50,0 \%$ \\
\hline Se muestra insuficiente asimilación de contenidos por parte de los estudiantes. & $46,7 \%$ & $53,3 \%$ \\
\hline $\begin{array}{l}\text { Se muestra insuficiente cumplimiento en la consecución de destrezas planificadas } \\
\text { en el currículo de su asignatura. }\end{array}$ & $46,7 \%$ & $53,3 \%$ \\
\hline $\begin{array}{l}\text { ACTIVIDADES DE REPERCUSIÓN EN LA SALUD MENTAL, FÍSICA Y EL } \\
\text { USO ADECUADO DEL TIEMPO }\end{array}$ & SI & NO \\
\hline Existe conflicto sicológico en la adaptación a la nueva modalidad de aprendizaje. & $63,3 \%$ & $36,7 \%$ \\
\hline $\begin{array}{l}\text { Se generan problemas personales producto del cambio de modalidad como } \\
\text { insomnio, cambio de carácter, problema económico. Familiar. Social, etc. }\end{array}$ & $71,7 \%$ & $28,3 \%$ \\
\hline $\begin{array}{l}\text { Se generan problemas de salud como estrés, gastritis producto del cambio de } \\
\text { modalidad. }\end{array}$ & $71,7 \%$ & $28,3 \%$ \\
\hline $\begin{array}{l}\text { Se muestra la falta de organización del tiempo personal producto del cambio social } \\
\text { emergente. }\end{array}$ & $63,3 \%$ & $33,7 \%$ \\
\hline
\end{tabular}




\begin{tabular}{|l|l|l|}
\hline Se muestra influencia de las actividades familiares en las laborales. & $66,7 \%$ & $33,3 \%$ \\
\hline $\begin{array}{l}\text { Considera que hay una Incidencia negativa en el respeto de su tiempo por parte } \\
\text { de los actores educativos: Padres de familia y estudiantes al contacto tecnológico. }\end{array}$ & $75,0 \%$ & $25,0 \%$ \\
\hline
\end{tabular}

Fuente: Los Autores (2020).

Se pudo observar en cuanto a la adaptación y apoyo al proceso de cambio que, del total de encuestados un 45\% afirman haber estado preparados para enfrentar el cambio de modalidad, al contrario de quienes no lo estuvieron debido a factores analizados como: un $65 \%$ indican la falta de equipo tecnológico sea este hardware o software, un $70 \%$ así mismo alegan la falta de capacitación, un $78,3 \%$ que señalan como insuficientes las políticas impuestas por los organismos gubernamentales y un porcentaje considerado bajo del $25 \%$ de personal que ve como, insuficiente el apoyo por parte de la institución donde laboran y así mismo el 30\% considera insuficientes a las políticas informáticas por parte de las autoridades académicas institucionales.

En referencia a la dimensión nombrada como alfabetización y destrezas en la comunicación virtual, se muestran como focos de interés que; un $35 \%$ anota tener bajo conocimiento en el uso de software de video conferencias que es la base para la consecución del hecho académico virtual, esto conlleva a que el $51,7 \%$ del total encuestado afirma tener insuficiente conocimiento del manejo de software de trabajo colaborativo, así como del conocimiento y aplicación de herramientas web 2.0 como por ejemplo; el 53,3\% no conoce el desarrollo de presentadores en línea, el 58,3\% no utiliza herramientas para colaboración sincrónica, el 56,7\% no aplica software para evaluación en línea y el $86,7 \%$ no usa pizarras digitales. Todo ello se notaría como parte de las consecuencias de que el $20 \%$ dice tener dificultades de expresión frente a la cámara de video, siendo un resultado preocupante que el 45\% muestra dificultades al mantener la comunicación con los estudiantes al otro lado de la línea desembocando en un 33,3\% afirmado como insuficiente control 
OAI-PMH: http://www.indteca.com/ojs/index.php/Revista_Scientific/oai

Artículo Original / Original Article

disciplinario con los estudiantes durante las conferencias.

Por otro lado, y en consecuencia con la distribución de servicios de ancho de banda contratados en los hogares y, la consecuente comunicación en línea, aseguran estar satisfechos con los planes de servicio el $71,7 \%$, destacándose además en este tema el impacto al $88,3 \%$ de los docentes por la cantidad de usuarios en el hogar frente al ancho de banda contratado, aunque el $58,3 \%$ afirman que son suficientes los servicios ofrecidos por las empresas proveedoras del servicio.

De los resultados conseguidos como cumplimiento de objetivos en el proceso de enseñanza-aprendizaje y que influyen directamente con el impacto en el proceso académico, se indica por parte del $15 \%$ de los docentes una falta de contenido científico suficiente para dictar sus clases, falencias en la suficiencia de material de apoyo didáctico expuesto por el $33,3 \%$ en coincidencia con la insuficiencia de material académico en relación con la asignatura que se imparte notado por el $33.3 \%$ de los docentes encuestados. La mitad de los docentes encuestados, $50 \%$, presentan una dificultad en el avance de los contenidos en función con la planificación vigente y esto conlleva también a afirmarse por parte de un $46,7 \%$ que existe una insuficiente asimilación de contenidos por parte de los estudiantes y por lo tanto señalan el $46,7 \%$, el incumplimiento en la consecución de destrezas planificadas en el currículo de la asignatura.

Se debieron además considerar parámetros como el análisis de actividades de repercusión en la salud mental, física y el uso adecuado del tiempo notándose que el $63,3 \%$ de docentes indican que existe conflicto sicológico en la adaptación a la nueva modalidad de aprendizaje que han conllevado al $71,7 \%$ de los mismos a la generación de problemas personales producto del cambio de modalidad como insomnio, cambio de carácter, problema económico, familiar, social, etc., y así mismo, coinciden un $71,7 \%$, que se han producido, problemas de salud tales como estrés, gastritis producto 
del cambio de modalidad.

Cabe indicar además que en base a los resultados, se muestra en un $63,3 \%$ de docentes, la falta de organización del tiempo personal producto del cambio social emergente, lo que ha influenciado en el $66,7 \%$, la falta de dosificación y mezcla de actividades familiares con las laborales que además producen el fenómeno de una Incidencia negativa en el respeto de su tiempo por parte de los actores educativos: Padres de familia y estudiantes a través del contacto tecnológico en el $75 \%$ de los docentes en la muestra de estudio.

\section{Conclusiones}

El impacto en la gestión docente generado luego del análisis expuesto, ha sido considerado como alto por un $63,3 \%$ de todos los encuestados y resulta consecuente con el impacto en el proceso académico, resultando un porcentaje similar del $61,7 \%$, producto del cambio a la modalidad virtual

Se puede notar que el cambio producido, producto de la pandemia Covid-19 en los procesos académicos ha sido inminente y la adaptación al mismo depende de factores como la alfabetización docente y su esfuerzo diario en procesos de capacitación en medios tecnológicos para estar acordes a las necesidades de los nuevos estudiantes, lo que Camacho (2018), los llama la generación Z, pues:

[...] Estos nativos digitales para comunicarse utilizan entornos digitales, les gusta la sinergia y el trabajo colaborativo, la finalidad es romper el paradigma de la educación 1.0 tradicional, dar el salto cualitativo a la educación 2.0 constructivista hasta llegar a la educación 3.0 o conectivista que es la era digital que permita enfrentar los retos de la sociedad educativa moderna (pág. 23).

Es necesario recomendar entonces el uso de herramientas web 2.0 de trabajo colaborativo en red, herramientas de gamificación, manejo de plataformas virtuales, entre otros factores, pero además se debe considerar el 
OAI-PMH: http://www.indteca.com/ojs/index.php/Revista_Scientific/oai

\section{Artículo Original / Original Article}

apoyo imperecedero que deben tener los actores educativos por parte de los organismos estatales de control, las autoridades de cada institución y la comunidad educativa que los rodea. Siendo conscientes del rol del docente virtual y sus capacidades frente a la crisis vivenciada, es preciso proponer el uso de varias herramientas de uso libre que la web facilita y que han sido referentes en capacitaciones y procesos educativos utilizados por distintas instituciones y personas inmersas en el campo académico.

Figura 1. Rueda de atributos básicos sugerida para un docente virtual.

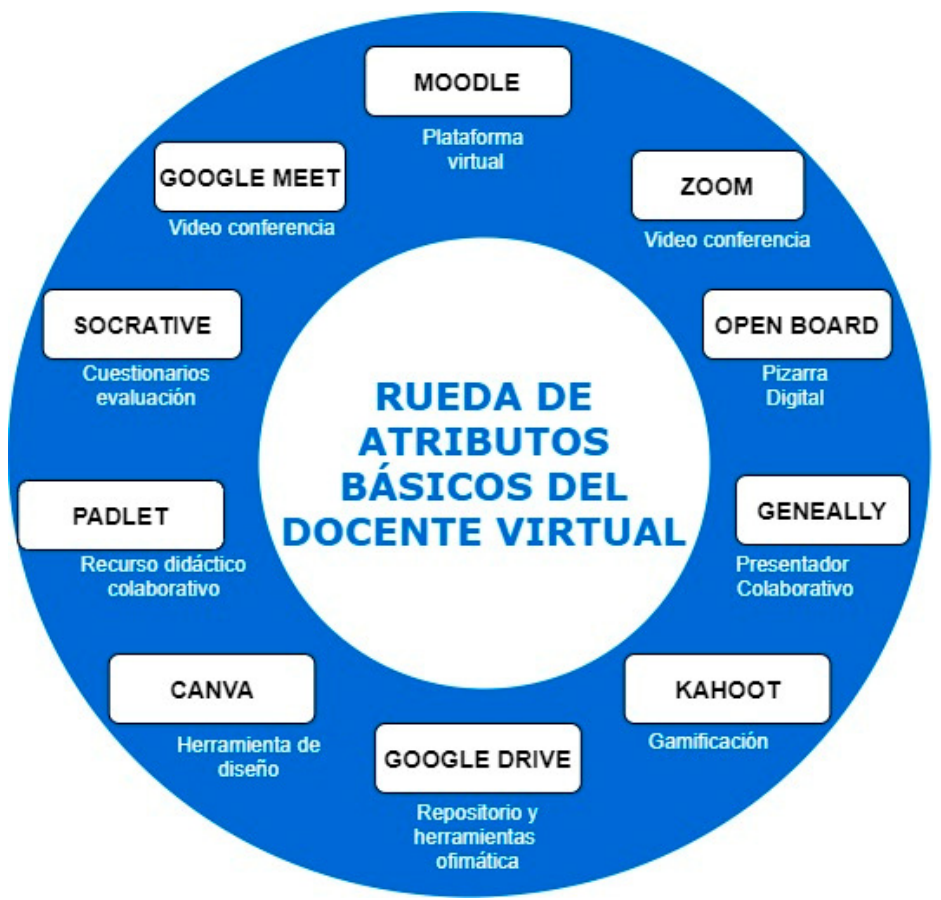

Fuente: Los Autores (2020).

Por ejemplo, la plataforma Moodle se la utiliza como base en un Entorno Virtual de Aprendizaje (EVA), en diferentes instituciones de Latinoamérica y el Ecuador con todas sus aplicaciones sincrónicas y asincrónicas. En caso de no disponer de este recurso, se sugiere el uso de software especializado de la web 2.0, mencionando en primer lugar al software de video conferencia Zoom y el Google Meet, entre los mejores posicionados en el medio académico; 
herramientas de trabajo colaborativo como el muro digital de Padlet; los presentadores gráficos y herramientas de diseño como el Genially y Canva; una pizarra digital residente que se mantiene en auge denominada OpenBoard; y herramientas de ofimática lideradas por Google Drive; la gamificación a través del Kahoot! y software que permite evaluar los procesos en línea con cuestionarios múltiples como el Socrative. Con lo expuesto se propone la denominación de una rueda de atributos básicos para un docente virtual y que sintetizan algunas herramientas como se puede observar en la figura 1.

Las herramientas que se mencionan tienen fácil acceso, cuentan con manuales de uso muy sencillo y que son de gran apoyo a la hora de sentarse frente a un computador e impartir una clase. Además, varias de ellas permiten el trabajo colaborativo en virtud de que el estudiante no es un ente pasivo, sino que debe participar del proceso a través de un adecuado manejo de sus emociones en coincidencia con el uso de metodologías activas que Rodríguez, García y Fuentes (2020), mencionan:

Se reafirma la relación existente entre el contexto y la experiencia personal de cada individuo, así como sus capacidades de autocrítica, regulación emocional y autoconocimiento en el proceso cognitivo, generando como consecuencia la optimización y revitalización de la enseñanza en cualquier espacio educativo (pág. 232).

Cada persona es un mundo de aprendizajes, producto de su crecimiento a través de la influencia de los espacios educativos utilizados, sus sentimientos, emociones, actitudes, aptitudes y desarrollo de destrezas adquiridas en todos los procesos de formación a los que le toco enfrentarse en el trayecto de su vida personal y profesional.

\section{Referencias}

Angarita, C., \& Cabrera, K. (2000). El corazón del rendimiento académico. 
OAI-PMH: http://www.indteca.com/ojs/index.php/Revista_Scientific/oai

Artículo Original / Original Article

Psicología desde el Caribe, (5),1-29, e-ISSN: 0123-417X. Recuperado

de: https://www.redalyc.org/articulo.oa?id=21300502

Caballero, C., Abello, R., \& Palacio, J. (2007). Relación del y el rendimiento académico con la satisfacción frente a los estudios en estudiantes universitarios. Avances en Psicología Latinoamericana, 25(2), 98-111, e-ISSN: 1794-4724. Recuperado de:

http://www.scielo.org.co/pdf/apl/v25n2/v25n2a7.pdf

Camacho, C. (2018). Visión Teórica Humanística Educativa de la Generación Z 3.0 en Tiempos Complejos. Revista Scientific, 3(9), 2038, e-ISSN: 2542-2987. Recuperado de:

https://doi.org/10.29394/Scientific.issn.2542-2987.2018.3.9.1.20-38

Castellanos, E., \& Castro, J. (2018). Aproximación Teórica para el uso de los Entornos Virtuales en el Proceso de Aprendizaje de los Estudiantes Universitarios. Revista Scientific, 3(7), 99-120, e-ISSN: 2542-2987. Recuperado de:

https://doi.org/10.29394/Scientific.issn.2542-2987.2018.3.7.5.99-120

Dolores-Martínez, M., \& Chávez, D. (2015). Plataforma tecnológica construcción de contenidos digitales para un posgrado virtual. Ra Ximhai, 11(4), 369-379, e-ISSN: 1665-0441. Recuperado de:

https://www.redalyc.org/articulo.oa?id=46142596027

Flores, D., \& Garrido, J. (2019). Competencias digitales para los nuevos escenarios de aprendizaje en el contexto universitario. Revista Scientific, 4(14), 44-61, e-ISSN: 2542-2987. Recuperado de: https://doi.org/10.29394/Scientific.issn.2542-2987.2019.4.14.2.44-61 Martínez, O., Martínez, O., Martínez, R., \& León, M. (2018). Comunicación para el Liderazgo en las Instituciones Educativas en el Contexto de la Complejidad. Revista Scientific, 3(9), 190-213, e-ISSN: 2542-2987. Recuperado de: https://doi.org/10.29394/Scientific.issn.2542$\underline{2987.2018 .3 .9 .10 .190-213}$ 
Martínez-Otero, V. (1997). Los adolescentes ante el estudio: Causas y consecuencias del rendimiento académico. ISBN: 84-245-0756-8. Madrid, España: Editorial Fundamentos.

Rodríguez, M., García, W., \& Fuentes, C. (2020). Valores éticos y emociones desde el desarrollo de metodologías activas en la formación docente. Revista Scientific, 5(15), 229-246, e-ISSN: 2542-2987. Recuperado de: https://doi.org/10.29394/Scientific.issn.2542$\underline{2987.2020 .5 .15 .11 .229-246 ~}$ 
OAI-PMH: http://www.indteca.com/ojs/index.php/Revista_Scientific/oai

Artículo Original / Original Article

\section{César Raúl Méndez Carpio \\ e-mail: cmendezc@ucacue.edu.ec}

Nacido en Cuenca, Ecuador, el 22 de febrero del año 1971.

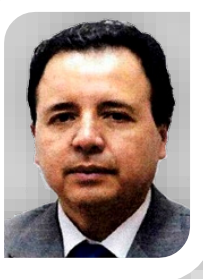

Dr. En Ciencias de la Educación, especialidad Ordenadores en la Universidad Católica de Cuenca (UCACUE); Magister en Docencia y Currículo para la Educación Superior en la Universidad Técnica de Ambato (UNITA); Magister en Administración de Empresas en la Universidad del Azuay (UDA); Actualmente me desempeño como Jefe de Investigación en el Colegio Militar "Abdón Calderón" y como docente en la Universidad Católica de Cuenca (UCACUE), en las Asignaturas: Estadística Aplicada, Tecnologías de Información y Comunicación. 


\section{Natalia Adriana Palacios Montero}

e-mail: npalacios@napalogistic.com

Nacida en Cuenca, Ecuador, el 21 de septiembre del año

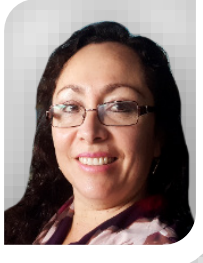

1977. Me gradué en el Colegio Manuela Garaicoa de Calderón, en la especialidad de Secretariado Español; soy Auditora de normas BASC para el capítulo Azuay; Actualmente me desempeño como General Manager en la Empresa NAPALOGISTIC IFF, que es un Freight Forwarders o Agente de Carga Internacional. 